\title{
Type of Investigation Not Yet Determined
}

National Cancer Institute

\section{Source}

National Cancer Institute. Type of Investigation Not Yet Determined. NCI Thesaurus.

Code C139464.

Details to determine the type of investigation are not yet available, but are being sought.

Do not use this code if the investigation is complete. 